\title{
Hybridverfahren zur EMV-Analyse elektrischer Leitungen über geschlitztem Grund
}

\author{
J. R. ter Haseborg, H.-D. Brüns, and H. Singer \\ Technische Universität Hamburg-Harburg, Germany
}

Zusammenfassung. Die Betrachtung niedrig geführter elektrischer Leitungen über leitendem Grund stellt besondere Anforderungen an die numerische Feldanalyse. Insbesondere für Fälle ungleichförmiger Leitungsführung oder ungleichförmigen Grunds werden Verfahren benötigt, die eine effektive EMV-Analyse zulassen. Die Verwendung von volldiskretisierten Modellen erfordert aufwändige Diskretisierungen, große Ressourcen und hohe Rechenzeiten. Daher werden Möglichkeiten gesucht, die effektive Leitungstheorie auf Anordnungen anzuwenden, deren direkte Berechnung in klassischer Betrachtungsweise nicht möglich ist. In der vorliegenden Arbeit wird ein Hybridverfahren vorgestellt, um den Einfluss von Schlitzen unterhalb von Leitungen in einer approximativen EMV-Analyse zu untersuchen. Hierzu dient neben der Leitungstheorie zur Berechnung des Leiterstroms eine momententheoretische Simulation auf Basis von magnetischen Linienströmen.

\section{Einleitung}

Die Simulation elektronischer Komponenten ist heutzutage ein unverzichtbarer Bestandteil der Systementwicklung geworden. Steigende Komplexität und der Betrieb bei höheren Frequenzen erfordern bereits vor der Fertigung eines Prototypen eine EMV-Analyse durch Simulationen, um ausreichende Störfestigkeit und begrenzte Störaussendung zu gewährleisten. Problematisch gestaltet sich bei der numerischen Simulation von Systemen insbesondere die Modellierung. Auf Grund sehr kleiner Querschnittsgeometrien von Leitungen und durchaus mehrere Größenordnungen über den Querschnittsabmessungen liegenden Gesamtabmessungen sind Rechenverfahren, die eine Volldiskretisie-

Correspondence to: J. R. ter Haseborg

(janremmer@terhaseborg.de)

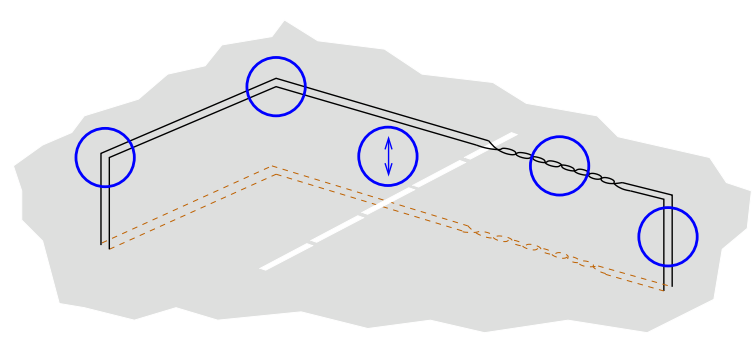

Bild 1. Ungleichförmig geführte Doppelleitung.

rung benötigen, Grenzen durch die zur Verfügung stehenden Ressourcen gesetzt.

Eine produktive Systemanalyse erfordert daher den Einsatz hybrider Verfahren. Je nach Anordnung kann es von entscheidender Bedeutung sein, bis zu welchem Grad die Interaktion der Teilmodelle untereinander im Rahmen des Hybridverfahrens berücksichtigt werden muss bzw. berücksichtigt werden kann. Bei der Untersuchung von über metallischem Grund geführten Leitungen bietet sich die Verwendung der klassischen Leitungstheorie zur Berechnung von Strömen und Spannungen in einem verteilten Netzwerk an. Sollen jedoch Effekte wie Abstrahlung oder Interaktion mit Diskontinuitäten in der Simulation berücksichtigt werden, muss die klassische Leitungstheorie erweitert oder mit anderen Verfahren gekoppelt werden.

Bei der Leitungsführung über metallischem Grund treten im Allgemeinen verschiedene Ungleichförmigkeiten auf, die in Abb. 1 vereinfacht dargestellt sind. Hierzu gehören z.B. horizontale und vertikale Knicke in Leitungen, vertikale Anschlüsse an leitenden Grund, ungleichförmige Leitungsführung (z.B. Verdrillung, Durchhang) und gesplitteter Grund (ggf. mit Punktschweißstellen). Im Rahmen dieses Beitrages wird die Berechnung von Leitungen über geschlitztem metallischem Grund vorgestellt. 


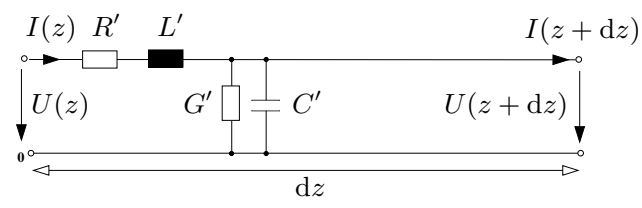

Bild 2. Leitungstheoretisches Ersatzschaltbild eines infinitesimal kleinen Leitungselements $d z$ einer Einfachleitung.

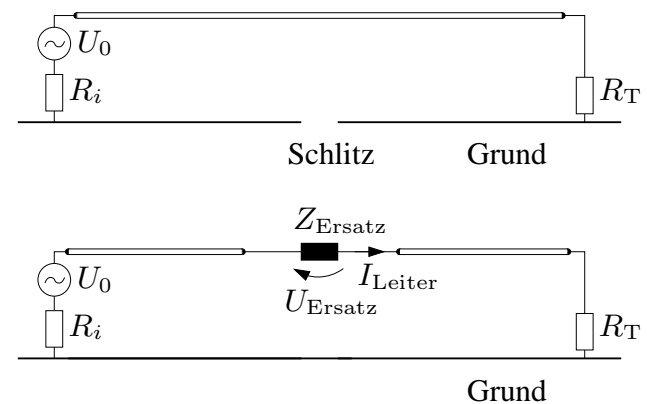

Bild 3. Berücksichtigung eines Schlitzes im Ersatzschaltbild.

\section{Grundlagen}

Im vorliegenden Verfahren werden die Leitungsströme zunächst über klassische Leitungstheorie ermittelt. Hierfür sind die notwendigen Parameter (Leitungsbeläge) $C^{\prime}, L^{\prime}, R^{\prime}$ und $G^{\prime}$ (siehe Abb. 2) zu bestimmen. Für kanonische Anordnungen lassen sich die Kapazitätsbeläge analytisch ermitteln, während im allgemeinen Fall eine Berechnung mit Hilfe eines 2D-MoM-Verfahrens Verwendung findet (Gebele, 2000). Der Induktivitätsbelag ergibt sich gemäß $\left[L^{\prime}\right]\left[C^{\prime}\right]=\mu \epsilon[1]$. Die endliche Leitfähigkeit des Leitermaterials wird über $\kappa_{\text {Leiter }}$ und den vorliegenden Leiterquerschnitt unter Berücksichtigung des Skineffekts in $R^{\prime}$ sowie die Leitfähigkeit einer dielektrischen Leiterummantelung in den Leitwertsbelag $G^{\prime}=\kappa_{\text {Isolation }} / \epsilon \cdot C^{\prime}$ eingebracht.

Die Interaktion von Leitung und Schlitz kann im Ersatzschaltbild durch eine komplexe Last $Z_{\text {Ersatz }}$ approximiert werden (siehe Abb. 3). Ein volldiskretisiertes Modell der hier betrachteten Anordnungen zeigt Bild 4. Um die Größe des komplexen Lastelements zu ermitteln, muss zunächst die Wirkung der Leitung auf den Schlitz betrachtet werden. Hierbei findet das Äquivalenzprinzip (Jordan, 1968) Anwendung, d.h., es wird statt eines Schlitzes in einer unendlich ausgedehnten elektrisch ideal leitenden Ebene ein magnetisch ideal leitfähiger Stab mit einem äquivalenten Durchmesser $d$ angenommen. Der Leiterstrom oberhalb des Schlitzes führt im Schlitz zu einem Magnetfeld $H_{\text {tan,Leiter (vgl. Bild 6), das sich }}$ näherungsweise über den analytischen Zusammenhang

$H_{\text {tan,Leiter }}=\frac{I_{\text {Leiter }}}{2 \pi h_{\text {Leiter }}}$

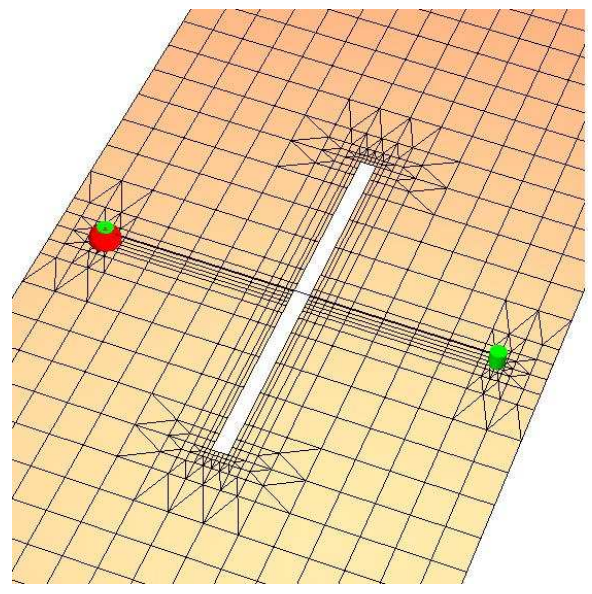

Bild 4. Volldiskretisiertes Modell eines über einen Schlitz geführten Leiters mit Generator (links) und Abschlusswiderstand (rechts).

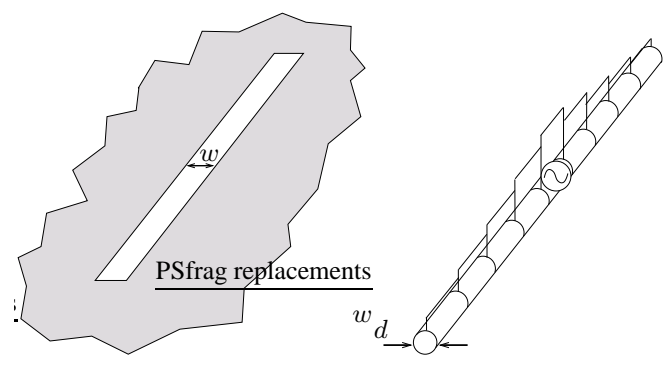

Bild 5. links: Schlitz in ideal leitender unendlich ausgedehnter Ebene, rechts: äquivalente Stabanordnung mit in Rechteckbasisfunktionen entwickeltem Magnetstrom.

beschreiben lässt. Das Linienintegral über das Magnetfeld des Leiters im Schlitz $H_{\text {tan }}$ liefert die magnetische Spannung $\theta_{\text {Schlitz }}$, mit der ein magnetischer Strom $K_{\text {Schlitz }}$ im Schlitz bzw. auf dem äquivalenten Stab angeregt wird:

$\theta_{\text {Schlitz }}=\int_{-s_{0}}^{s_{0}} H_{\tan } \cdot \boldsymbol{e}_{\varphi} \cdot \boldsymbol{e}_{\text {Schlitz }} \mathrm{d} s$.

Der sich auf dem Stab ausbildende magnetische Linienstrom wurde in Rechteckbasisfunktionen entwickelt und momententheoretisch ermittelt. Bild 5 zeigt die bei Annahme einer unendlich ausgedehnten elektrisch leitfähigen Ebene äquivalente Stabanordnung. Für den Durchmesser $d$ des äquivalenten Stabes gilt $d=w / 2$ (Leugner, 2004), wobei $w$ die Breite des Schlitzes darstellt.

Die Entwicklung des magnetischen Linienstroms $K$ ist äquivalent zur Bestimmung des elektrischen Stroms $I$ zu sehen.

Elektrische Streufeldstärke:

$$
\begin{aligned}
& \boldsymbol{E}(I)=\frac{1}{4 \pi} \int(-j \omega \mu I \boldsymbol{e}_{l^{\prime}} \frac{e^{-j k R}}{R} \\
&\left.-\frac{j}{\omega \epsilon} \frac{\partial}{\partial l^{\prime}} I \operatorname{grad} \frac{e^{-j k R}}{R}\right) \mathrm{d} l .
\end{aligned}
$$




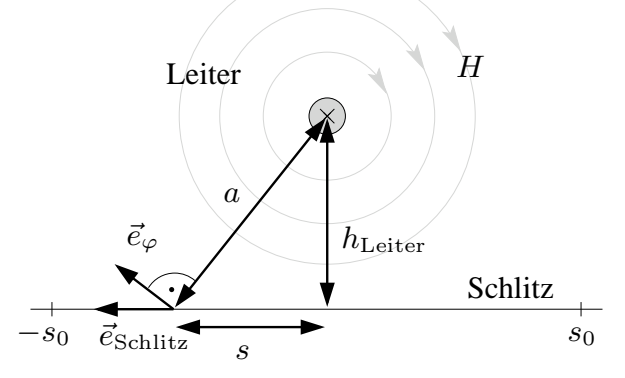

Bild 6. Leitungsquerschnitt im Bereich des Schlitzes.

Magnetische Streufeldstärke:

$$
\begin{aligned}
& \boldsymbol{H}(K)=\frac{1}{4 \pi} \int(-j \omega \epsilon K \boldsymbol{e}_{l^{\prime}} \frac{e^{-j k R}}{R} \\
&\left.-\frac{j}{\omega \mu} \frac{\partial}{\partial l^{\prime}} K \operatorname{grad} \frac{e^{-j k R}}{R}\right) \mathrm{d} l .
\end{aligned}
$$

Die durch den angeregten Schlitz auf die Leitung zurückwirkende elektrische Feldstärke lautet näherungsweise:

$E_{\text {tan,Schlitz }}=\frac{K_{\text {Schlitz }}}{2 \pi h_{\text {Leiter }}}$.

Das Linienintegral über $E_{\text {tan }}$ ergibt nun die Spannung $U_{\text {Ersatz }}$, die über dem die Wirkung des Schlitzes berücksichtigenden Ersatzelement $Z_{\text {Ersatz }}$ abfallen muss:

$U_{\text {Ersatz }}=\int_{-s_{0}}^{s_{0}} E_{\text {tan }} \cdot \boldsymbol{e}_{\varphi} \cdot \boldsymbol{e}_{\text {Leiter }} \mathrm{d} s$.

Da der Leiterstrom $I_{\text {Leiter }}$ bekannt ist, lässt sich das Ersatzelement $Z_{\text {Ersatz }}$ über

$Z_{\text {Ersatz }}=\frac{U_{\text {Ersatz }}}{I_{\text {Leiter }}}$

bestimmen und gemäß Bild 3 in das Ersatzschaltbild einfügen, um durch erneute leitungstheoretische und netzwerktheoretische Rechnung den Strom im Leiter über dem Schlitz in einer weiteren Iteration zu bestimmen. Dieser veränderte Strom führt zu einem veränderten Magnetstrom im Schlitz sowie einer veränderten Rückwirkung auf die Leitung, so dass ein iteratives Verfahren entsteht. Weicht der ermittelte Strom im Leiter über dem Schlitz nur noch geringfügig vom in der vorhergehenden Iteration bestimmten Strom ab, wird die Iteration abgebrochen und $Z_{\text {Ersatz }}$ gilt als bestimmt. Für jede Frequenz lässt sich der iterative Algorithmus damit wie folgt schreiben:

$$
\begin{gathered}
\text { Leitungstheorie: Bestimmung des Stroms } I_{\text {Leiter }}^{(i)} \\
\Downarrow \\
\begin{array}{c}
\text { Berechnung der Schlitzanregung } \theta_{\text {Schlitz }} \\
\Downarrow
\end{array}
\end{gathered}
$$

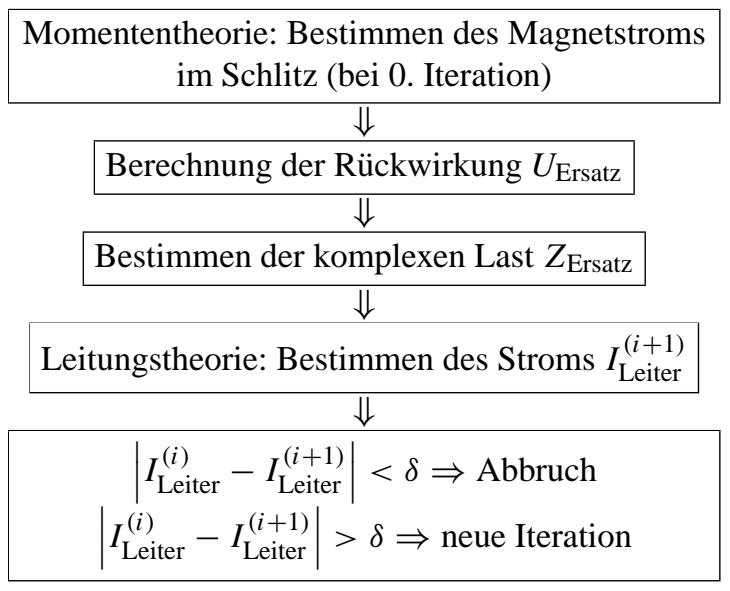

Die Rückwirkung des Schlitzes auf die Leitung kann auch auf einem anderen Rechenweg durch eine Betrachtung der der Leitung entnommenen Leistung $S$ berücksichtigt werden. Mit Hilfe der Eingangsimpedanz des äquivalenten magnetischen Dipols (vgl. Bild 5, rechts)

$Z_{\text {ein }}=\frac{\theta}{K}$

lässt sich die Leistung, die der Leitung entnommen wird, als

$S=\frac{\theta^{2}}{Z_{\text {ein }}}$

angeben. Das im Ersatzschaltbild zu berücksichtigende komplexe Lastelement bestimmt sich damit zu

$Z_{\text {Ersatz }}=\frac{S}{I_{\text {Leiter }}^{2}}$.

Simulationen haben gezeigt, dass beide Verfahren bei entsprechend ausreichender numerischer Integration nach Gl. 6 zum selben Ergebnis führen.

\section{Simulationsergebnisse}

Das oben beschriebene Verfahren wurde implementiert und durch vergleichende MoM-Simulationen verifiziert. Drei ausgewählte Beispiele werden im folgenden Abschnitt vorgestellt.

\subsection{Einfachleitung über Schlitz}

Das erste Beispiel (Bild 7) besteht aus einer $1 \mathrm{~m}$ langen Leitung, die in $8 \mathrm{~mm}$ Höhe mittig über einen $2 \mathrm{~m}$ langen und $5,8 \mathrm{~mm}$ breiten Schlitz geführt wird. Bild 8 zeigt den Strom am Ende der Leitung ohne Schlitz (über idealem Grund) in einer MoM-Simulation und in einer Simulation auf Basis der Leitungstheorie. Auf Grund der geringen Höhe der Leitung stimmen die Resultate der klassischen Leitungstheorie und der MoM-Simulation hier gut überein, da der Leistungsverlust durch Abstrahlung auf dem $1 \mathrm{~m}$ langen Leitungsstück 


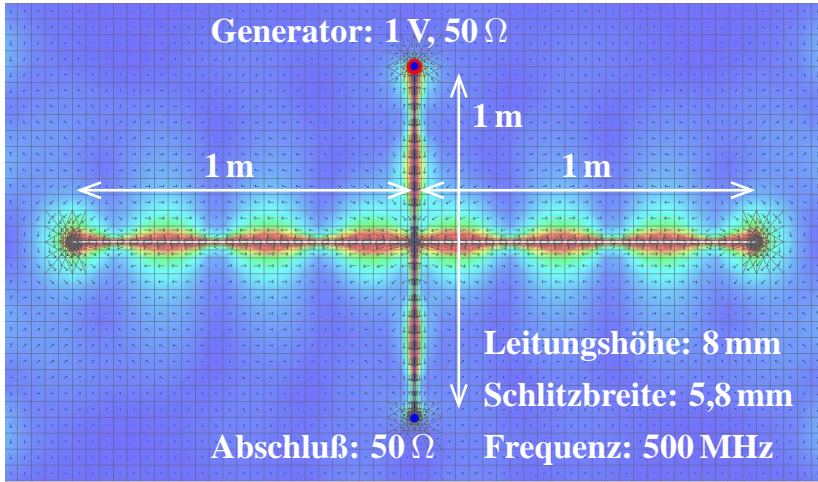

Bild 7. Aufsicht auf eine Leiter-Schlitz-Anordnung; der Schlitz (Länge: $2 \mathrm{~m}$ ) ist horizontal und der Leiter (Länge: $1 \mathrm{~m}$ ) vertikal dargestellt.

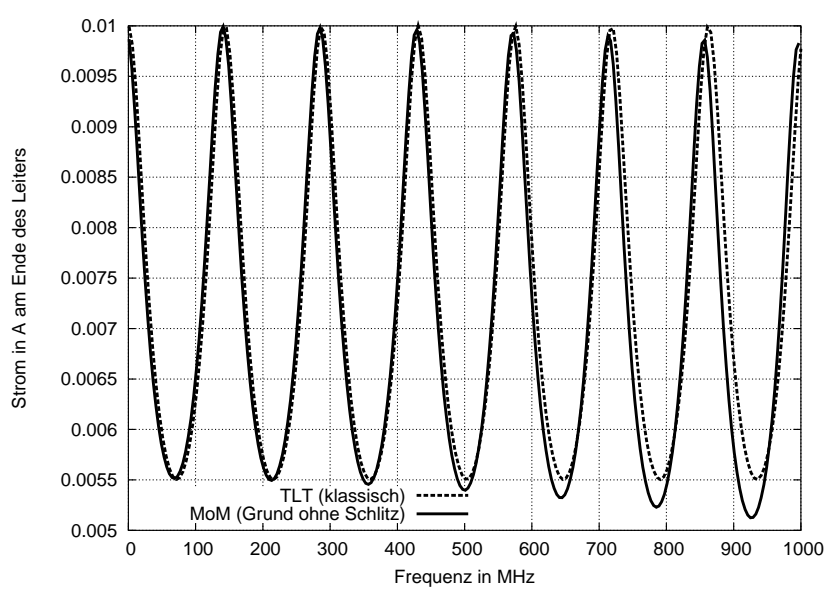

Bild 8. Strom im Abschluss einer $1 \mathrm{~m}$ langen Leitung (Höhe: $8 \mathrm{~mm}$ ) über idealem Grund.

zunächst vernachlässigbar ist. In Bild 9 wird deutlich, dass bei Berücksichtigung des Schlitzes der Stromverlauf am Ende der Leitung erheblich von der Simulation ohne Schlitz abweicht. Findet das oben beschriebene Verfahren Anwendung, so lässt sich der Einfluss des Schlitzes, wie in Bild 10 gezeigt, bis ca. $800 \mathrm{MHz}$ sehr gut wiedergeben.

\subsection{Doppelleitung über Schlitz}

Im zweiten Beispiel werden zwei Leitungen im Abstand von $50 \mathrm{~cm}$ über einen Schlitz geführt (siehe Bild 11). Auf Grund der geringen Höhe von $8 \mathrm{~mm}$ und dem grossen Abstand von $50 \mathrm{~cm}$ findet ohne Anwesenheit des Schlitzes keinerlei Verkopplung statt. Die zwei weit auseinanderliegenden Leiter der Doppelleitung werden leitungstheoretisch korrekt als Doppelleitung simuliert, so dass die Kopplung zwar berücksichtigt würde. Bild 12 bestätigt allerdings, dass bei dem im Verhältnis zur Höhe großen Abstand der Leiter voneinander keine Verkopplung erkennbar ist. Unter Verwendung

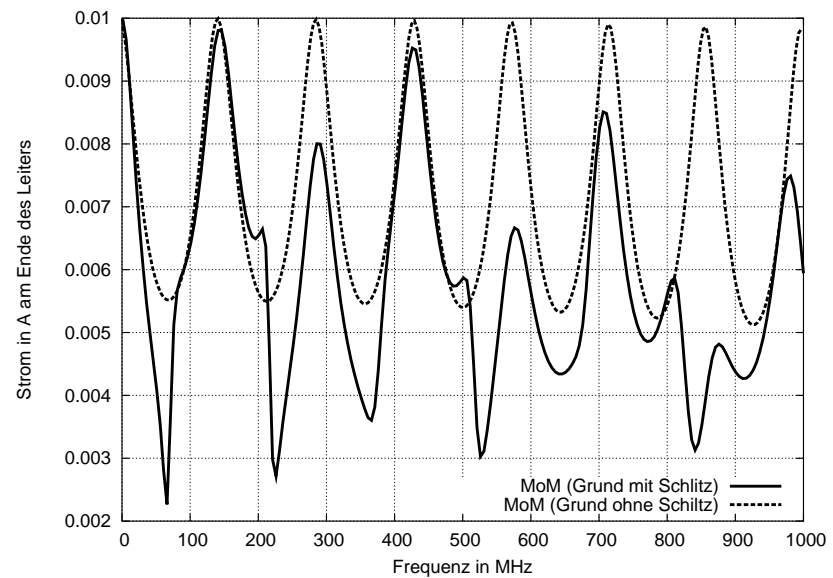

Bild 9. Strom im Abschluss einer $1 \mathrm{~m}$ langen Leitung (Höhe: $8 \mathrm{~mm}$ ) über nicht geschlitztem bzw. geschlitztem Grund nach Bild 7.

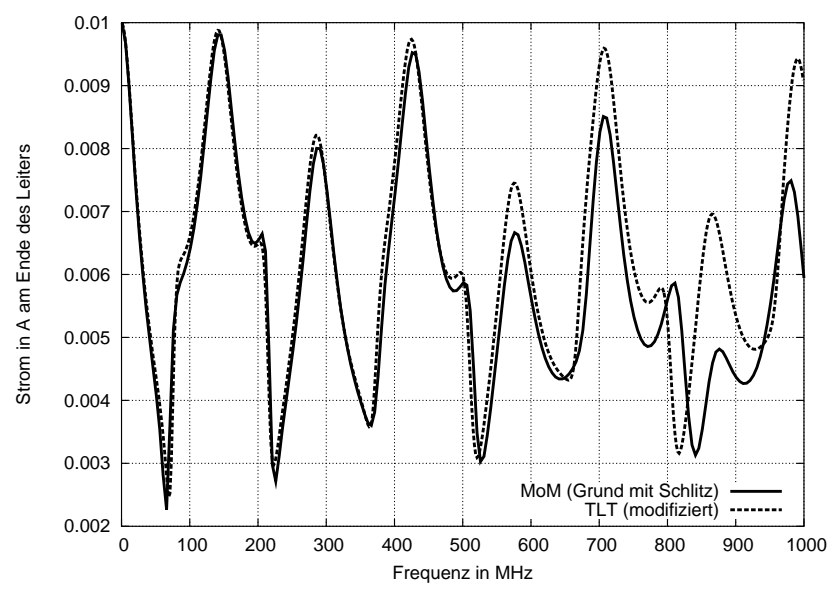

Bild 10. Strom im Abschluss einer $1 \mathrm{~m}$ langen Leitung (Höhe: $8 \mathrm{~mm}$ ) über geschlitztem Grund nach Bild 7.

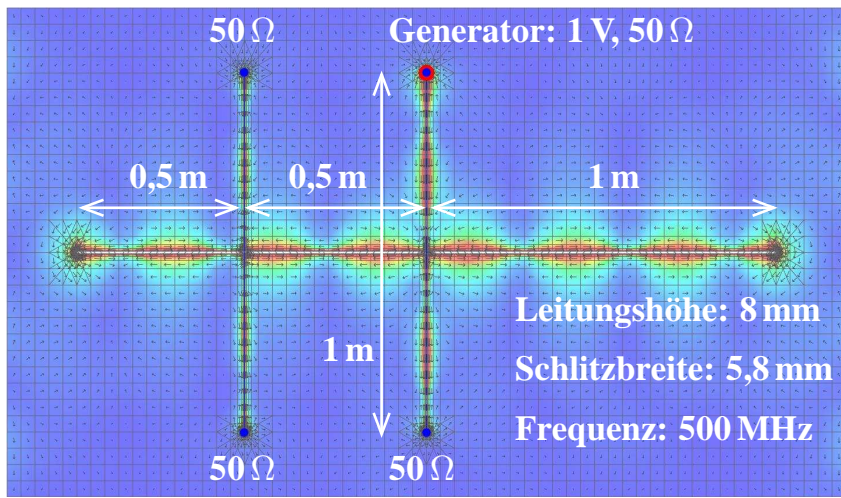

Bild 11. Aufsicht auf eine Doppelleiter-Schlitz-Anordnung; der Schlitz (Länge: $2 \mathrm{~m}$ ) ist horizontal und die Leiter (Länge: $1 \mathrm{~m}$ ) sind vertikal dargestellt.

der modifizierten Leitungstheorie, wie sie im Rahmen dieser Arbeit vorgestellt wird, kann bis ca. $800 \mathrm{MHz}$ auch in 


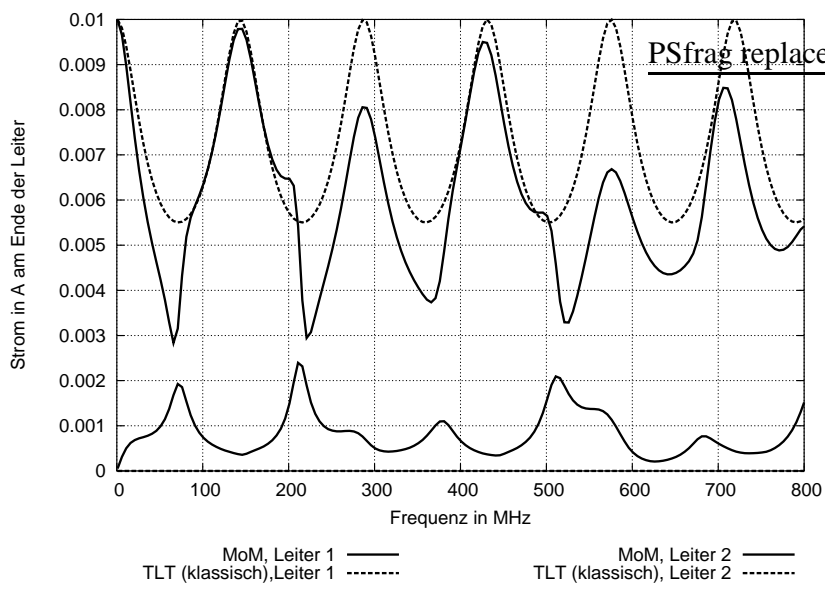

Bild 12. Ströme in den Abschlüssen der in Bild 11 dargestellten Anordnung berechnet mit MoM und klassischer Leitungstheorie (die oberen Kurven zeigen jeweils die Ströme am Ende des gespeisten Leiters 1).

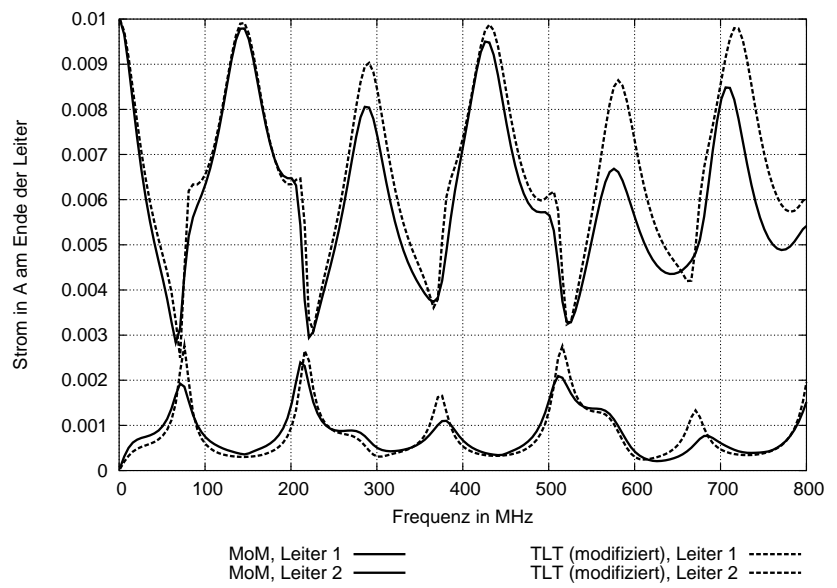

Bild 13. Ströme in den Abschlüssen der in Bild 11 dargestellten Anordnung berechnet mit MoM und erweiterter Leitungstheorie (die oberen Kurven zeigen jeweils die Ströme am Ende des gespeisten Leiters 1).

diesem Beispiel eine gute Übereinstimmung mit der MoMVollsimulation erreicht werden (siehe Abb. 13). Bei dieser Simulation wurde nur die Anregung des Schlitzes durch die gespeiste Leitung berücksichtigt und der Einfluss des Schlitzes auf die Leiter auf zwei entsprechende Ersatzimpedanzen im Zug beider Leiter abgebildet. Die Wirkung der nicht gespeisten Leitung auf den Schlitz wurde hierbei vernachlässigt. Die Überkopplung auf die nicht gespeiste Leitung, die nur durch den Schlitz stattfindet, liegt in auffallend guter Übereinstimmung mit dem MoM-Vollmodell.

\subsection{Einfachleitung über unterbrochenem Schlitz}

Im dritten Beispiel wird eine Leitung oberhalb eines an zwei Stellen unterbrochenen Schlitzes simuliert (Bild 14).

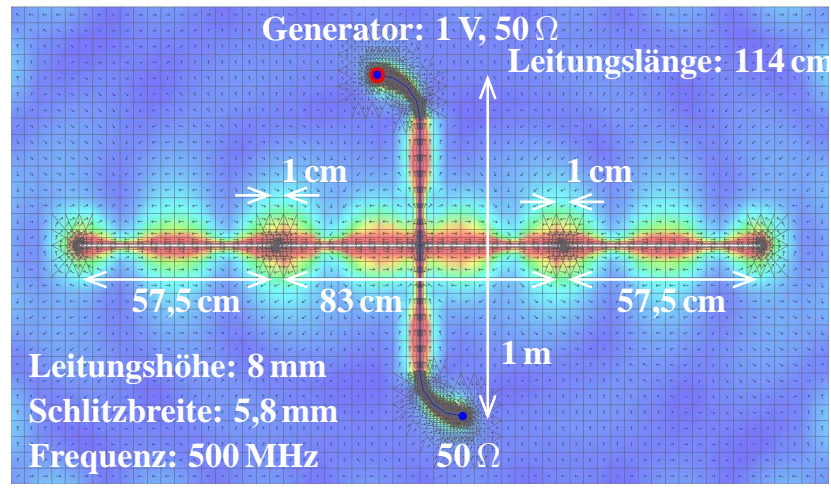

Bild 14. Aufsicht auf eine Leiter-Schlitz-Anordnung; der durch Punktschweißstellen unterbrochene Schlitz (Gesamtlänge: $2 \mathrm{~m}$ ) ist horizontal und der an den Enden leicht abgewinkelte Leiter (Länge: $1,14 \mathrm{~m}$ ) vertikal dargestellt.

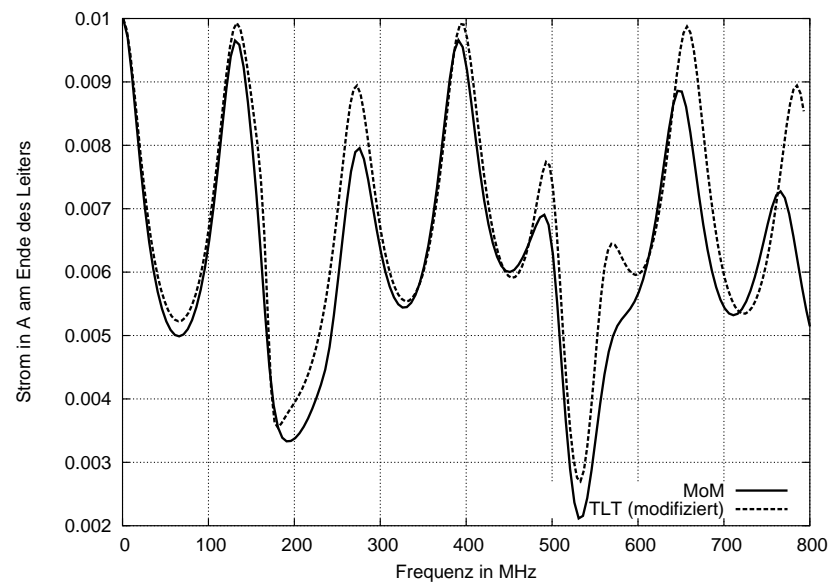

Bild 15. Strom im Abschluss einer 1,14 m langen Leitung (Höhe: $8 \mathrm{~mm}$ ) über mehrfach geschlitztem Grund nach Bild 14 .

Die MoM-Referenzsimulation zeigt hierbei im Vergleich zum nicht unterbrochenen Schlitz im ersten Beipiel einige Veränderungen in der Stärke der Resonanzpeaks (siehe Bild 15). Die Simulation mit Hilfe der modifizierten Leitungstheorie zeigt jedoch auch in diesem Fall eine gute Übereinstimmung mit dem Verlauf der Referenzkurve. Lediglich die absolute Höhe einiger Resonanzen wird im Fall der modifizierten Leitungstheorie überschätzt.

\section{Zusammenfassung}

Das vorgestellte Hybridverfahren zur Erweiterung der Leitungstheorie auf Leitungen, die über Schlitze geführt werden, zeigt in den dargelegten Beispielen eine gute Übereinstimmung mit den MoM-Referenzkurven. Mit steigender Frequenz auftretende Abweichungen in der Amplitude der mit Hilfe des Hybridverfahrens ermittelten Ströme werden auf die Vernachlässigung der dreidimensionalen Feldverhält- 
Tabelle 1. Rechenzeiten der Momententheorie.

\begin{tabular}{lcc} 
Modell & \multicolumn{2}{c}{ MoM } \\
\cline { 2 - 3 } & Unbek. & parallel, 4 Knoten \\
\hline Einfachleitung & 5190 & $120 \mathrm{~s} /$ Freq. \\
Doppelleitung & 6026 & $180 \mathrm{~s} /$ Freq. \\
unterbrochener Schlitz & 8664 & $480 \mathrm{~s} /$ Freq.
\end{tabular}

nisse im Kreuzungspunkt von Leitung und Schlitz zurückgeführt. Bei der Bewertung der Ergebnisse bleibt weiterhin zu berücksichtigen, dass der Schlitz in unendlich ausgedehnter Ebene modelliert und mögliche Abstrahleffekte durch Plattenresonanzen vernachlässigt werden. Die in den betrachteten Modellen geringe Abstrahlung der horizontalen Leitungsabschnitte über dem Grund wird ebenfalls vernachlässigt. Ein grosser Vorteil des Hybridverfahrens sind die geringe Rechenzeit und die geringen Ressourcen, die zur Simulation der zur Diskussion stehenden Anordnungen notwendig sind. Die Tabellen 1 und 2 zeigen die unterschiedlichen Rechenzeiten von reinen MoM-Simulation und Hybridverfahren auf. Auf Grund der Modellierung der Anordnungen in Leitungstheorie und Netzwerktheorie entfallen aufwändige dreidimensionale Strukturmodelle und deren Diskretisierung. Ein weiterer Vorteil gegenüber dem Referenzverfahren ist die Anwendung auf Mehrfachleitungen und sehr niedrig geführte Leitungen, bei denen die 'wideseparation approximation' (Leitungshöhen und Leitungsabstände kleiner als der fünffache Leiterradius) nicht eingehalten wird und somit eine MoM-Simulation mit Linienleiterapproximation nicht mehr möglich ist.
Tabelle 2. Rechenzeiten des Hybridverfahrens.

\begin{tabular}{lc} 
Modell & $\begin{array}{c}\text { Hybridverfahren } \\
\text { skalar }\end{array}$ \\
\hline Einfachleitung & 0,5 s/Freq. \\
Doppelleitung & 0,5 s/Freq. \\
unterbrochener Schlitz & 0,5 s/Freq.
\end{tabular}

\section{Literatur}

Gebele, O. and Brüns, H.-D.: Ein Hybridverfahrten zur Berechnung von Leitungseinkopplung im Rahmen der Oberflächenstromsimulation, in: Kleinheubacher Berichte, 359-366, 2000.

Jordan, E. C. and Balmain, K. G.: Electromagnetic Waves and Radiating Systems, Prentice-Hall Series, 2 edn., 1968.

Leugner, D.: Berechnung der elektromagnetischen Einkopplung durch Aperturen mit analytischen Verfahren und der Momentenmethode (Dissertation), Fortschritt-Berichte VDI, Reihe 21, Nr. 360 (ISBN 3-18-336021-7), Technische Universität Hamburg-Harburg, 2004. 